\title{
Simulation of Data Communication System taking into Account Dynamic Properties
}

\author{
Galina M. Antonova \\ Vadim V. Makarov \\ The faculty of Cybernetics, National Research Nuclear University MEPhI, Moscow, Russia, https : / / mephi . ru/ \\ Trapeznikov Institute of Control Sciences ICS RAS, Russian Academy of Sciences, Moscow, Russia, \\ gmanteipu.ru, makfonedipu.ru
}

\begin{abstract}
This paper continues the study, presented at the 8th EUROSIM Congress on Modeling and Simulation and devoted to creation of algorithm and simulation model of network functioning, taking into account dynamic characteristics of the network in condition of variable relationship signal-to-noise. Simulation algorithm was augmented for adequately representation of the state of the real network i.e. possible changes of topology due to the link failures and disabling individual nodes. It is possible to expand the capabilities of the model presented in the 8th Congress as a simulation model of Information Flow on Transport Layer of Open System Interconnection Model. The current version of the model realizes input of the adjacency matrix describing the network topology, the algorithm of the path search by Dijkstra on the network level, and simulation of the loss of connection. So the main goal of the new paper is to bring the structure of the model to the structure of the real network and to check the possibility of transferring a given amount of information in conditions of interference by means of evaluation of coefficient of readiness for Data Communication System.
\end{abstract}

Keywords: modeling, Monte-Carlo simulation, information technologies, algorithm

\section{Introduction}

Modeling of the dynamic properties of the Data Communication network is one of the urgent tasks in the modern theory of communication. Processes of data transmission have very high speed and the data transfer equipment includes specialized devices that provide perform the necessary calculations at speeds far exceeding the speed of imitation of processes of data transmission in modern software environments. At the same time, quite often in practice there is a need to evaluate the number of criteria of quality that are related not only internal properties of hardware and data transmission protocols but external integral characteristics.

They are related to external characteristics of the ne twork as a whole or its separate fragments. These characteristics usually are interested for the creators of information systems. One of the main criteria of quality is the availability of Data Communication System (DCS). The other important criterion is the probability of message delivery in the presence of noise. These criteria help to check the properties of the fragment of network or the network as a whole and test its suitability for the solution of the problem of the transfer of large amounts of information at any given time.

The throughput for different channels of network and communications centers and the procedures of changing network topology must be realized as part of the overall procedure of simulation of DCS for evaluating dataflow and network capacity in system as a whole. Thus the paper deals with a problem of construction of adequate simulation model of DCS taking into account dynamic processes in separate channels and nodes of system as a whole. Usually dynamic processes are emerged in form of various violations of the network topology, i.e. in node failures, in communication failures in the communication channels, in the packet loss and in the receiving an increased number of errors due to the increase of the level of interference in communication channels. A universal tool must be created for research and testing of various algorithms of network operation and for correction of new protocols at the stage of preproject inspection.

The structure of paper is as follows. Introduction demonstrates some distinctive features of the statement of the problem. The section 2 is devoted to describing of a static variant of the simulation model. It contains brief analysis of results of imitation experiments. In the section 3 algorithm of simulation of DCS with variable topology and quick details about modeling computer's program are described. The section 4 involves simulation results for checking proposed algorithm.

For a formal definition of DCS designed for the transmission of big volume of information represented in a form of graph structure, we can use the definition in the form of a functional signed graph from (Shul'tsc, Kulba et al, 2011). A tuple of parametric functional graph $<(X, E), V, W, U>$ is introduced. In this recording 
$G=(X, E)-$ directed graph, describing the structure of DCS, symbol $V-$ a set of parameters of the vertices of the graph, where every vertex is according to node of network. Parameters of the vertices must define all characteristics of nodes necessary for modeling of DCS and evaluating its' criteria of quality. For example, parameters define procedure of modeling host-router, involved in the selection of the direction of information transmission, or node - repeater designed only to enhance the signal to increase the communication distance. $V=\{\operatorname{Pnode}(x), x \in X\}$, where $\operatorname{Pnode}(x)-\mathrm{a}$ set of parameters of the vertex (node) $x$. Symbol $U$ describes the parameter space, a $\mathrm{W}$ - weights of edges, simulating communication channels. Weights of edges can change during modeling, and finding the path for message transfer.

The main directions of modeling of DCS in modern theory of communication involve three different approaches.

First of all modeling of the propagation medium of the signal allows investigating new principles of design of equipment for communication. Mathematical and statistical models help to create estimates of main characteristics of communication technique for data transferring. They help to define requirements to new equipment. Such models are described in (Proakis, 1995; Rappaport, 2002; Saleh, Valenzuela, 1987; Spencer, Rice et al, 1997) and they expand possibilities of designing of new DCS.

Secondly the modeling of the functioning of DCS as complicate technical system opens new directions for optimization and improvement of existing constructions of DCS. A huge number of models are created over the years of development of the theory of communication. These include works (Foschini, 1996; Antonova, 2007), devoted to data transmission in condition of fading, and many other publications. For example, a set of works are the well-known (Irvine, Harle, 2001; Tanenbaum, 1996) and others.

Thirdly special direction is devoted to different modern problems. At the moment models for describing of various indicators of quality of service (QoS) have been actively developed. For example, for the transfer of information via packets the set of basic criteria involve Bandwidth, Delay, Packet loss and Jitter. A significant number of modern publications including the Russian-speaking illuminate this direction.

\section{Algorithm of Simulation of Data Communication System}

\subsection{Statement of Simulation Problem}

The problem of modeling the networks' dynamic properties may be solved by means of imitation statistical model of DCS, proposed in (Antonova, 2013; Antonova, Titov, 2011; Antonova, Kolutcsky,
2015). DCS is represented as a set of channels and communication centers. Large centers for data gathering create a set of signal office centers. In common with channels for information transferring they create graphical image of network topology with variable structure. In the existing networks information may be transmitted in different directions. So almost every node can be both a starting and an end node in the procedure of message delivery to the addressee. This fact determines dynamic changes in the network.

In order to simplify debugging and simulation procedures the assumption is introduced that data is transmitted in one direction from one initial vertex to the destination vertex in network fragment under consideration. This assumption simplifies the development of simulation algorithms, but it is a limitation in the development of a universal instrument for studying processes of transferring large amounts of data in DCS. However imitation statistical model will allow checking quality of data transferring by means of imitation experiments for dynamic stochastic DCS according to algorithm from (Antonova, 2013; Antonova, Titov, 2011; Antonova, Kolutcsky, 2015).

The proposed statement of the problem for simulation of dynamical features of DCS is considered in detail in (Antonova, Titov, 2011). The first variant of a simulation model for the static mode of operation and single state of network topology is presented in flow chart of simulation algorithm in Figure 1. It does not use the possibilities of modern software tools and focused on the tedious procedure of the source data preparation for modeling. Symbol $S$ represents the number of message. Symbol $S k$ is labeling maximal quantity of messages under simulation.

Matrix $M K(I J)$ contains symbol 1 on position with number of row $I$ (node with number $I$ ) and column with number $J$ (channel with number $J$ ) if such channel exists in network topology. Symbol Jmax defines maximal quantity of channels, outgoing from current node with number $I$ in accordance with network topology. Symbol $I k$ is maximal quantity of nodes.

Symbol $J t$ is a number of channel selected in simulation procedure according to special criterion. This channel connects the current node with node $I(J t)$ in accordance with network topology.

After start of algorithm the necessary input data is read from a file. The movement of the first message from the first node to next node according the topology of fragment of network under consideration begins with the scanning of outgoing channels. All outgoing channels are described in matrix $M K(I J)$. This matrix is continuously visible in the search of the outgoing path.

Simulation for every outgoing channel is fulfilled for transfer time evaluation. The level of errors for given signal-to-noise ratio or for given imitation model of transfer process is also determined. The channel is selected to send a message when the cycle of viewing 
for channels leaving the current vertex is over. Selection criteria may be different.

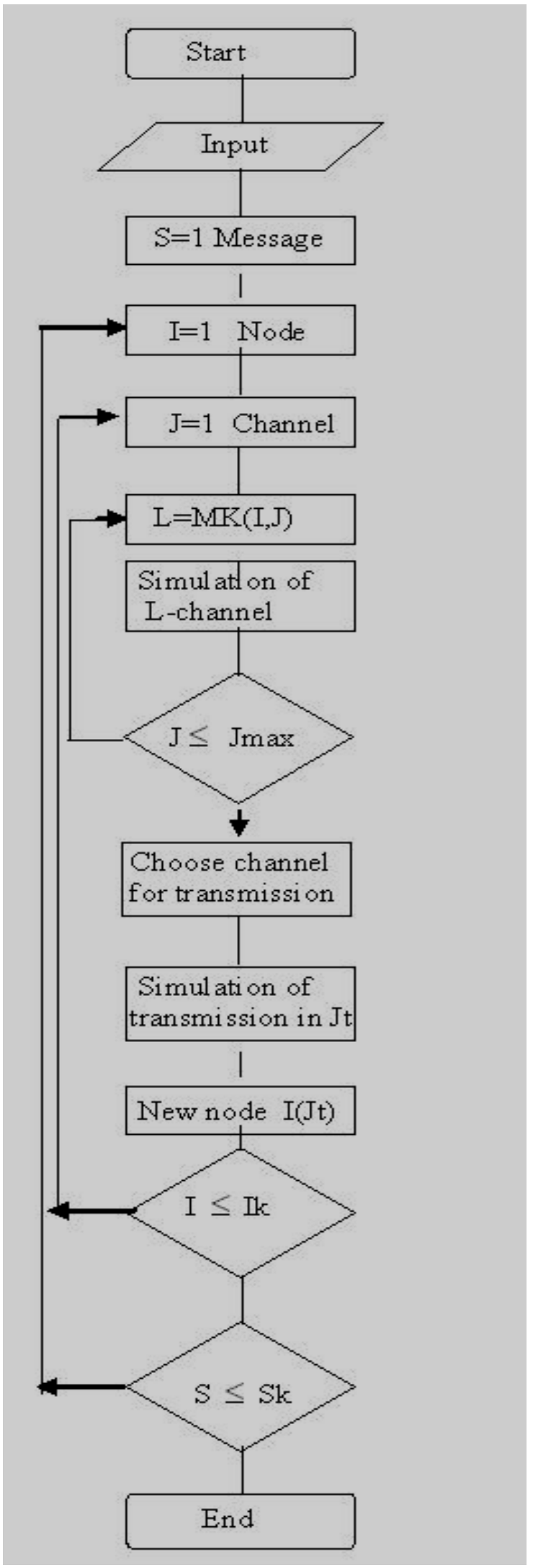

Figure 1. Flow Chart of a static variant of the simulation model.
After the end of the simulation of the transfer process new node is fixed. It will be that vertex of the graph in which the movement of the first message will stop. For new node all procedures for choice and simulation of the transfer process in the channel will be repeated. Thus the first message will continues the moving to final vertex of the graph. When message arrives in final vertex $I k$, the number of message is tested. If transferring of information didn't over the next message is selected.

Further the moving of the following message will be simulated since first node and so on. At the end of list of messages when last message with number $S k$ will arrive in final node the availability coefficient will be calculated by means of definition of ratio of time of useful moving of messages along fragment of network to common time of simulation of the transfer process. This value is saved in special variables.

This scheme will be supplemented with the outer loop managing random changes in network topology under conditions of noise in functioning of communication channels and nodes. Such model will reflect the variable structure of the computer network in the DCS, i.e. the possibility of link failure or the blocking nodes of a communication network, and subsequent recovery as a result of urgent repair. It will allow checking the impact of interference on the delivery time of messages in the network. This model allows to estimate the availability of the network and to provide additional opportunities for evaluation of the possibility of Big Data accumulation.

\subsection{The first results of modeling}

The first variant of modeling program was realized by means of Pl-language and ES 1045 computer. It allowed evaluating transmission characteristics of the information flow for static mode of operation of the network under interference. This model did not take into account the variable structure of the network under real conditions of information transferring but was used in imitation experiments for testing and evaluating networks characteristics in noise conditions (Antonova, 1996; Antonova, 1999; and Antonova, 2007).

\section{Simulation of Data Communication System with Variable Topology}

The considered variant of simulation model of DCS was raw realization of proposed simulation algorithm by means of $\mathrm{C}++$ language and personal computer. It created the basis for detailed implementation of the model reproducing the dynamic structure of data transferring network (Antonova, 2013; Antonova, Titov, 2011; Antonova, Kolutcsky, 2015). The flow chart of a new simulation algorithm is shown in Figure 2. Additional symbol $N t$ in this figure defines the 
number of variant of network topology under consideration in simulation procedure. New variant of topology may be appearing only for new message. It limited models possibilities but it was necessary for testing simulation algorithm. New topology may involves the channel interference, the communication gap between individual nodes and the shutdown of the node, reducing noise, the restoration of the link between nodes, the repair of nodes.

A new variant of the simulation model is implemented in the environment of Microsoft Visual Studio 13. The developed algorithm simulates the process of transferring messages within the network fragment which is represented as oriented weighted graph. The vertices of the graph, i.e. the network nodes, simulated work of the packet switches via the TCP/IP Protocol with realization of procedures of determining the optimum route of messages transmission.

Edges of the graph simulate the communication channels with a certain bandwidth determined by the current value of the ratio signal-to-noise. In input data for simulation model the network graph is interpreted as the adjacency matrix, each element of which records the presence or absence of communication between nodes in network.

For adaptation to possible changes in the real network such as communication gap, a node failure, and addition of the node with specified links, an adjacency matrix reflecting the network topology duplicates the matrix allowing for program user to dynamically track the topology changes in the process of simulation program functioning.

Weights of connections between network nodes are defined by the relative time of messages' delivery between nodes according to the ratio, which varies with the changes in noise power:

$$
T=1 / C,
$$

where $C$ - throughput capacity of communication lines that are installed dynamically from the known ratio of the $\mathrm{C}$. Shannon:

$$
C=F \log \left(1+P_{S} / P_{S h}\right),
$$

where $F$ - is the bandwidth of the communication line, $P_{S}$ - signal power, $P_{S h}$ - noise power.

A minimal message delivery time is selected as a criterion for choice an optimal route for messages transmission between the start and end nodes of the network in the simulation model.

Messages are transferred between the specified fixed network nodes (start and end nodes). At each vertex for determination the channel for transmission the optimal route to the destination node is searched and for transmission the first link of the found route is selected.

For the next point of the route the optimal route to the destination network node is searched again and so on until the end node will be reached. Finding of the optimal route based on the algorithm, which is used famous Dijkstra's algorithm.

Each message from the stream in the process of the simulation model functioning appears in the window indicating the route to the destination node and the relative time of motion.

The following objects are defined in proposed algorithm: the array of distances between vertices; active vertex; dynamic list of available vertices from the active vertex (the list of visited vertices). The dimension of the array of distances corresponds to the rank of the adjacency matrix. Each element of the distances array is a structure. It includes the distance from the initial vertex to the vertex corresponding to the element of the array with current number (the initial distance is infinity) and the list of vertices making the route from the initial vertex to the considered vertex from the array (at the initial moment the list is empty).

The active vertex is special variable. It contains number of the adjacency matrix row when the search algorithm fulfills definition of outgoing channels. The list of visited vertices is dynamical array. According algorithm vertices connected with active vertex are added in that list and visited vertices are deleted. At the beginning of the algorithm initial node is an active vertex. The list of vertices making the route for first element of the array of distances will contain initial node and the array of distances from initial node will contain zero. The optimal route search procedure involves following stages: the adjacency matrix row associated with active vertex is examined; adjacent vertices are defined and added in the end of the list of visited vertices. The node having visited vertices is deleted from the top of the list. The node, which became the first in the list, converts to active vertex. The distance from initial vertex to vertex associated with active vertex is defined. If it is less than distance fixed in the array of distances early, than both distance and route in the array of distances are corrected.

The algorithm ends when the list of visited vertices is over. The criterion for the optimal route search is value of the message delivery time. The optimal route is defined with using matrix contained values reverse of throughput of networks channels. These values are created automatically in procedure of algorithm realization. The relationship

$$
T p=1 / \log \left(1+P_{S} / P_{S h}\right),
$$

defines value of each existing connection.

Value $T p$ is formed by means of simulation of the signal-to-noise relationship. Different distribution laws known from the results of statistical studies of DCS may be used for describing the signal-to-noise relationship. The average value of the signal-to-noise relationship is chosen equal to 100 . The system timer controls changes in topology. It is the lack of simulation model. 


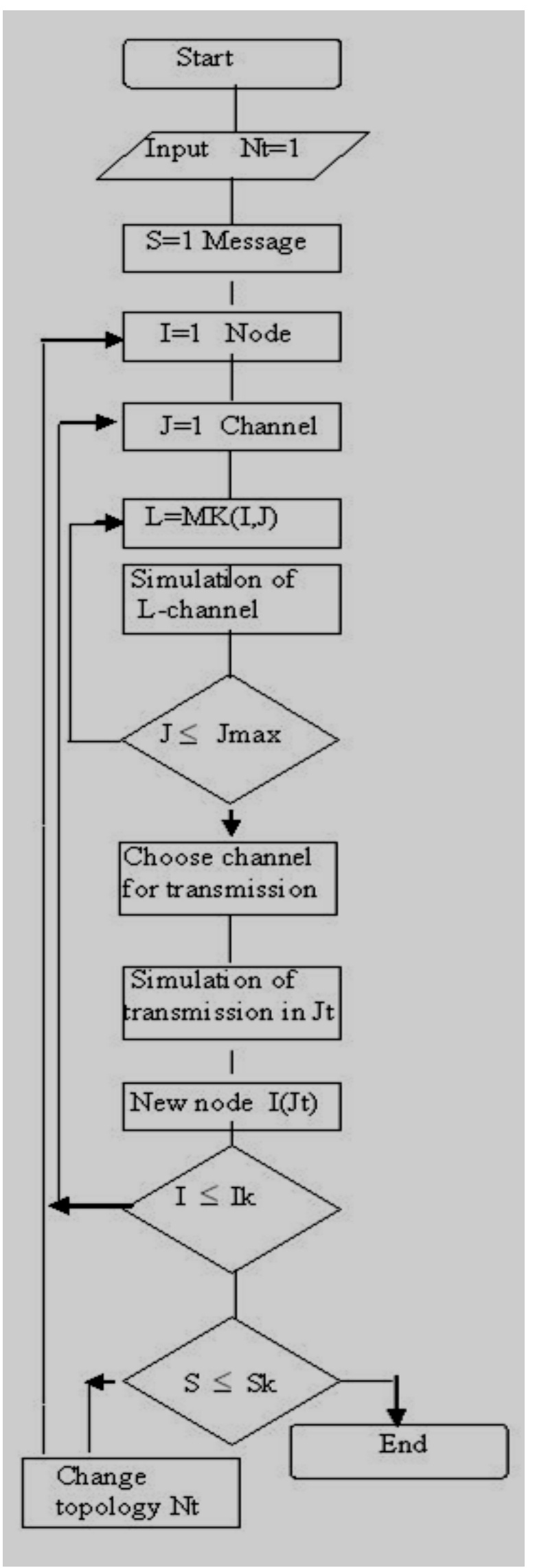

Figure 2. Flow Chart of a dynamic variant of the simulation model.
Every message from information stream appears in the window of simulation model. The route to final node and time of motion are demonstrated in window. All modeling events are fixed in track of events according to order of their receipt.

All changes to the network structure dynamically adjusted in the adjacency matrix. Because of calls to the adjacency matrix are happen from different threads in a model the mutual exclusion of these flows is organized. To do this a synchronization object "mutex" is used. This object is installed in a special signaling state if not busy by any thread. This object at any point in time can only hold one thread that prevents simultaneous access to a shared resource.

\section{Results of Simulation Experiments}

Initial network topology is selected in the form shown in the Figure 3 . The adjacency matrix $M$ describing this network fragment will sets by user of program in special Windows Forms with the use of element of control Data Grid View.

$$
M=\left(\begin{array}{llllllllll}
1 & 1 & 1 & 0 & 0 & 0 & 0 & 0 & 0 & 0 \\
0 & 0 & 0 & 1 & 1 & 0 & 0 & 0 & 0 & 0 \\
0 & 0 & 0 & 0 & 1 & 0 & 0 & 0 & 0 & 0 \\
0 & 0 & 0 & 0 & 1 & 1 & 0 & 0 & 0 & 0 \\
0 & 0 & 0 & 0 & 0 & 0 & 1 & 0 & 0 & 0 \\
0 & 0 & 0 & 0 & 0 & 0 & 1 & 1 & 0 & 0 \\
0 & 0 & 0 & 0 & 0 & 0 & 0 & 1 & 1 & 0 \\
0 & 0 & 0 & 0 & 0 & 0 & 0 & 0 & 0 & 1 \\
0 & 0 & 0 & 0 & 0 & 0 & 0 & 0 & 0 & 1 \\
0 & 0 & 0 & 0 & 0 & 0 & 0 & 0 & 0 & 1
\end{array}\right) .
$$

Weights of connections are established dynamically in the process of the functioning of simulation model. According to simulation algorithm experiment consists in transmission of message flow from the initial vertex of the network fragment to a final vertex of the network fragment. Channel interference, the link failure and disabling individual nodes are considered.

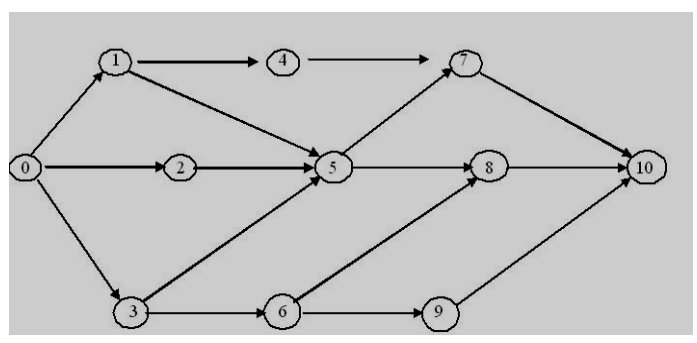

Figure 3. Scheme of a fragment of the communication network.

Results of simulation experiment are shown in Table I. The transmission of 30 messages in condition of change relationship signal-to-noise, the link failures and disabling individual nodes are considered. Table 1 contains results from Windows Forms: number of message (first column); time of message transmission 
(second column); the order of the passage of nodes from network fragment (third column); delivery information about messages (fourth column). Because of a long period of disconnection in the network fragment is simulated, the last three messages are not delivered.

\section{Conclusions}

A series of simulation results is only a checking of proposed algorithm. It needs in quality programming and extensive testing in terms of the input variable factors reflecting the structure of real networks. Big volume of initial input data are needed for creation of detailed and adequate estimates of performance and availability of DCS, but this problem is much easier then the problem of creating models of dynamic network for data transfer. The instruments for control of information flow in real network involve the repair of failed channels, addition of new channels, increasing of velocity of data transmission and extension of channel bandwidth by means of installation of new equipment and others. Further improvement of the model consists in the development of the structure of the program modules for simulation of different types of communication channels, support modules and the control program, taking into account the received results. Once the application package has been validated, the adequate results of simulation may forecast a situation in functioning of equipment of communication network under conditions of large load.

Table 1. Simulation results.

\begin{tabular}{|c|c|c|c|}
\hline \multirow{3}{*}{$\begin{array}{c}\text { Message } \\
1\end{array}$} & \multicolumn{3}{|c|}{ Characteristics of the motion } \\
\hline & \multirow{2}{*}{$\begin{array}{c}\text { Time } \\
1.015790 \\
\end{array}$} & \multirow{2}{*}{$\frac{\text { Route }}{14710}$} & Path of events \\
\hline & & & Start \\
\hline 2 & 1.226979 & 014710 & Start \\
\hline 3 & 1.101203 & 025810 & Start Channel 8-10 End \\
\hline 4 & $\underline{1.212878}$ & 036910 & Start \\
\hline 5 & $\underline{1.218719}$ & 035710 & Start Node 6 \\
\hline 6 & 1.197147 & 025710 & Start \\
\hline 7 & 0.992137 & 014710 & Start Channel 3-5 End \\
\hline 8 & 1.211201 & 015710 & Start \\
\hline 9 & 0.998856 & 015710 & Start \\
\hline 10 & 1.128747 & 015710 & Start Node 9 \\
\hline 11 & $\underline{1.116496}$ & 014710 & SStart Channel 1-5 End \\
\hline 12 & 1.170238 & 025710 & Start \\
\hline 13 & 1.124116 & 014710 & Start \\
\hline 14 & 1.362350 & 014710 & Start \\
\hline 15 & 1.245929 & 025710 & Start Channel 0-2 End \\
\hline 16 & 1.391276 & 014710 & $=$ Start Node 2 End \\
\hline 17 & 1.212394 & 014710 & Start \\
\hline 18 & 1.364307 & 014710 & Start \\
\hline 19 & $\underline{1.302780}$ & 014710 & Start Channel 5-8 End \\
\hline 20 & 1.294338 & 014710 & Start \\
\hline 21 & 1.138025 & 014710 & Start \\
\hline 22 & 1.382752 & 0147710 & Start \\
\hline 23 & 1.097262 & 014710 & Start Channel 5-7 End \\
\hline 24 & 1.273273 & 014710 & Start \\
\hline 25 & 1.382320 & 014710 & Start \\
\hline 26 & 1.104619 & 014710 & Start Channel 1-4 End \\
\hline 27 & & & Start A message is lost \\
\hline 28 & & & Start A message is lost \\
\hline 29 & & & Start A message is lost \\
\hline
\end{tabular}

\section{Acknowledgements}

Authors thank lecturer of Moscow Financial-Juridical University K. N. Kolutcsky for conscientious work as a programmer.

\section{References}

Galina M. Antonova. Realization of the Optimization Simulation Approach in the Selection of an Algorithm for the Functioning of Data Communication Systems. Automation and Remote Control, 57(9):1357-1363, 1996.

Galina M. Antonova. Choice of Noise Immune Correcting Codes by the $L P_{\tau}$-optimization within the Framework of the Optimization- Simulation Approach. Automation and Remote Control, 60(9): 1347-1352, 1999.

Galina M. Antonova. The mesh methods of uniform probe for investigation and optimization of the dynamical stochastic systems. Moscow: Phizmatlit. 2007.

Galina M. Antonova and A. P. Titov. Simulation of information flow in e-Governance. In Proc. 5-th AllRussian Science - practical Conference on Simulation and its Application in Science and Industry, St. Petersburg: OAS Center of Design and Shipbuilding. 5(1):325-328, 2011.

Galina M. Antonova. Simulation of Information Flow on Transport Layer of Open System Interconnection-Model. In Proc. of 8th EUROSIM Congress on Modelling and Simulation, Cardiff, Wales, UK: IEEE Press, Sep. 2013, pages $567-572$.

Galina M. Antonova and Konsyantin N. Kolutcsky. Simulation model of the data transfer process in the network segment. In Proc. 2-th International Science practical Conference on "Modern information technologies in professional activity”, Moscow: MFUA, 2: 15-18, 2015.

J. Irvine, D. Harle. Data Communications and Networks: An Engineering Approach. England: John Wiley\&Sons. 2001.

G. J. Foschini. Layered space-time architecture for wireless communication in a fading environment when using multiple antennas. Bell Labs Technical Journal, 1(2): 4159, 1996.

J. G. Proakis. Digital Communications. NY: McGraw Hill. 1995.

T. Rappaport. Wireless communications: principles and practice. New Jersey: Prentice Hall PTR. 2002.

A. Saleh, R. Valenzuela. A Statistical Model for Indoor Multipath Propagation. IEEE Journal on Selected Areas in Communications, 5(2): 128-137, 1987.

V. L. Shul'tsc, V. V. Kulba and others, Information control in condition of active confrontation: models and methods. Moscow: Science. 2011.

Q. Spencer, M. Rice, B. Jeffs, M. Jensen. A statistical model for angle of arrival in indoor multipath propagation, IEEE Vehicular Technology Conference. 47: 1415-1419, 1997.

A. S. Tanenbaum. Computer networks. Upper Saddle River, NJ: Prentice Hall. 1996. 